\title{
p53 protects lung cancer cells against metabolic stress
}

\author{
CHOMPUNOOT SINTHUPIBULYAKIT ${ }^{1,3}$, WANIDA ITTARAT ${ }^{3}$, \\ WILLIAM H. ST. CLAIR ${ }^{2}$ and DARET K. ST. CLAIR ${ }^{1}$ \\ ${ }^{1}$ Graduate Center for Toxicology, ${ }^{2}$ Department of Radiation Medicine, University of Kentucky, Lexington, \\ KY, USA; ${ }^{3}$ Faculty of Medical Technology, Mahidol University, Bangkok, Thailand \\ Received June 18, 2010; Accepted July 30, 2010
}

DOI: 10.3892/ijo_00000811

\begin{abstract}
The preferential use of aerobic glycolysis for energy production by cancer cells, a phenomenon known as the 'Warburg effect', is well recognized and is being considered for therapeutic applications. However, whether inhibition of glycolysis will be effective in all types of cancer is unclear. The current study shows that a glycolytic inhibitor, 2-deoxyD-glucose (2DG), exhibits the cytotoxic effect on non-small cell lung cancer in a p53-dependent manner. 2DG significantly inhibits ATP production in p53-deficient lung cancer cells (H358) but not in p53-wt cells (A549). In contrast to p53-wt cells, p53-defective cells are unable to compensate for their need of energy via oxidative phosphorylation (OXPHOS) when glycolysis is inhibited. In the presence of p53, increased ROS from OXPHOS increases the expression of p53 target genes known to modulate metabolism, including synthesis of cytochrome c oxidase 2 (SCO2) and TP53-induced glycolysis and apoptosis regulator (TIGAR). Importantly, 2DG selectively induces the expression of the antioxidant enzymes manganese superoxide dismutase (MnSOD) and glutathione peroxidase 1 (GPx1) in a p53-dependent manner. The results demonstrate that the killing of cancer cells by the inhibitor of glycolysis is more efficient in cancer cells without functional p53 and that p53 protects against metabolic stress by up-regulation of oxidative phosphorylation and modulation of antioxidants.
\end{abstract}

\section{Introduction}

Constitutively switching the main route of energy production from oxidative phosphorylation to aerobic glycolysis is a prominent hallmark of cancer cells $(1,2)$. A high glycolytic rate provides special advantages for proliferating cells. While aerobic glycolysis is an inefficient way to generate energy (ATP), glycolysis yields ATP at a faster rate compared to

Correspondence to: Dr Daret K. St. Clair, Graduate Center for Toxicology, University of Kentucky, College of Medicine, Lexington, KY 40536, USA

E-mail: dstc100@uky.edu

Key words: 2-deoxy-D-glucose, p53, cancer metabolism, oxidative stress oxidative phosphorylation to support the uncontrolled cell growth (3). The intermediates from the glycolytic pathway supply building blocks for biosynthesis of macromolecules, including NADPH, citrate and glycerol for lipids, and ribose sugars for nucleotides. In addition, excessive lactic acids from glycolysis favor tumor invasion and can be used by other cells $(4,5)$.

Recent findings suggest that tumor suppressor $\mathrm{p} 53$ plays a role in energy metabolism by regulating metabolic processes (6). p53 serves as an energy inspector sensing the decrease of ATP levels and stimulating oxidative phosphorylation through upregulation of the synthesis of the cytochrome $c$ oxidase 2 (SCO2) gene that encodes a copper chaperone protein required for the assembly of mitochondrial cytochrome $\mathrm{c}$ oxidase (complex IV) $(7,8)$, as well as transcriptional activation of subunit I of cytochrome c oxidase (9). Furthermore, p53 activates TP53-induced glycolysis and the apoptosis regulator (TIGAR), which functions to direct glucose to the pentose phosphate pathway (PPP), as well as glucose-6-phosphate dehydrogenase (G6PD), a glycolytic enzyme that catalyzes a rate-limiting step in the PPP $(10,11)$. The increase in PPP results in the stimulation of nucleotide synthesis and production of NADPH, which is an important component of the antioxidant defense system (12). In addition to the role of p53 in the regulation of mitochondrial respiration, p53 inhibits glycolysis by repressing the transcription of GLUT1, GLUT4 genes that encode glucose transporters (13) and the phosphoglycerate mutase $(P G M)$ gene that encodes a glycolytic enzyme responsible for the rearrangement of phosphoglycerate (14). Therefore, the inhibition of glycolysis and the induction of PPP and oxidative phosphorylation may mediate the tumor suppressive effect of p53.

2-Deoxy-D-glucose (2DG), a non-metabolizable glucose competitor, has been demonstrated to exert selective cytotoxic effects in a number of cancer cell lines but not in normal cells (15-18). Growth inhibition and apoptosis induction are among the mechanisms of 2DG-induced cytotoxicity $(19,20)$. Our previous work revealed that 2DG differentially kills non-small lung cancer cells with defective p53 status (21). However, the mechanisms by which p53 mediates the resistance of cancer cells to $2 \mathrm{DG}$-induced cytotoxicity is unknown. In this study, we show that p53 protects against 2DG-induced metabolic stress by enhancing oxidative metabolisms and inducing primary antioxidant enzymes. The results suggest that inhibition of glycolysis may be effective for advanced cancer with defective p53 status. 


\section{Materials and methods}

Cell culture conditions and treatments. Human non-small cell lung cancer cell lines, A549 and H358, were purchased from American Type Culture Collection (ATCC) and were cultured as previously described (21). All cells were free of mycoplasma contamination according to the test results performed monthly. 2-Deoxy-D-glucose (Sigma, MO, USA) was dissolved in $1 \mathrm{X}$ PBS, pH 7.4 and diluted in culture medium to the concentration specified for an experiment.

Glucose measurement. Cells were treated with $20 \mathrm{mM}$ 2DG for $3,6,12,24$, and $48 \mathrm{~h}$, after which the cultured medium was collected for glucose concentration measurement following the manufacturer's instructions (Human Gesellschaft fur biochemica und Diagnostica mbH, Wiesbaden, Germany).

ATP determination. Cells were treated as described above for glucose measurement. Cells were washed twice with ice-cold 1X PBS pH 7.4, collected and resuspended in PBS. Cells were homogenized for $30 \mathrm{sec}$ on ice. ATP level was measured in total cell homogenate using the ATP determination kit (Invitrogen, CA, USA)

Lactate determination. The A549 and H358 homogenates, prepared as described for assessment of ATP, were used to estimate the lactate level using the Lactate assay kit developed by Biomedical Research Service Center (NY, USA).

Mitochondrial respiratory complex I and complex II activities assay. Complex I and complex II specific activities were measured as previously described (22). Complex I specific activity was measured by following the decrease in absorbance due to the oxidation of NADH at $340 \mathrm{~nm}\left(\varepsilon 6.81 \mathrm{mM}^{-1} \mathrm{~cm}^{-1}\right)$. The activity was calculated by the differences in $\mathrm{dA} / \mathrm{min}$ with and without the complex I inhibitor, rotenone. Complex II activity was measured by following the reduction of 2,6dichlorophenolindophenol at $600 \mathrm{~nm}\left(\varepsilon 19.1 \mathrm{mM}^{-1} \mathrm{~cm}^{-1}\right)$ and calculated by the differences in $\mathrm{dA} / \mathrm{min}$ with and without ubiquinone.

Mitochondrial respiratory complex IV activity assay. Cells were washed twice with ice-cold PBS, collected and centrifuged. The pellets were used to determine complex IV activity according to the manufacturer's instructions (Invitrogen). The activity was determined colorimetrically by following the oxidation of reduced cytochrome c. The amount of the enzyme is measured by using a complex IVspecific antibody conjugated with alkaline phosphatase. The specific activities of complex IV were calculated as the ratio of mitochondrial complex IV activity/amount.

Intracellular reactive oxygen species (ROS) measurement. ROS measurement was performed using the oxidation-sensitive (5-[and -6]-carboxy-2,7-dichlorodihydrofluorescein diacetate; carboxy- $\mathrm{H}_{2}$ DCFDA) and oxidized carboxy-DCFDA fluorescent dye (Invitrogen). Following treatment, cells were washed with $\mathrm{PBS}$ and resuspended in PBS containing $1 \mathrm{mM}$ $\mathrm{CaCl}_{2}, 1 \mathrm{mM} \mathrm{MgCl}$, pH 7.4 (PBS/ $\mathrm{MgCl}_{2} / \mathrm{CaCl}_{2}$ ). Cells were incubated with $20 \mu \mathrm{M}$ of carboxy- $\mathrm{H}_{2}$ DCFDA or carboxy-
DCFDA at $37^{\circ} \mathrm{C}$ for $30 \mathrm{~min}$ in the dark; cells were then washed and covered with $\mathrm{PBS} / \mathrm{MgCl}_{2} / \mathrm{CaCl}_{2}$, and the fluorescent intensity was measured using the Gemini XPS fluorescence microplate reader (Molecular Device, CA, USA), at excitation $485 \mathrm{~nm}$ and emission $528 \mathrm{~nm}$. The fluorescent intensity in cells preloaded with carboxy- $\mathrm{H}_{2}$ DCFDA was normalized to that in cells preloaded with carboxy-DCFDA (ratio of $\mathrm{H}_{2}$ DCFDA/DCFDA) to control for the cell number, dye uptake, and ester cleavage differences between different treatment groups.

Mitochondrial superoxide measurement. The mitochondrial superoxides were measured using MitoSOX ${ }^{\mathrm{TM}}$ Red mitochondrial superoxide indicator (Invitrogen) according to the manufacturer's protocol. The fluorescences obtained from oxidation of MitoSOX reagents by superoxide were normalized to the number of cells.

mRNA isolation and real-time RT-PCR. Cells were collected and processed to obtain mRNA using the MagNA Pure Compact RNA Isolation Kit (Roche, IN, USA). Then, mRNA was used in the reverse transcription reaction Superscript ${ }^{\mathrm{TM}}$ III First-Strand synthesis system (Invitrogen). cDNA was used as the template for real-time RT-PCR with a set of human specific primers SCO2-forward, 5'-gcagcaaaagcgaacagaa-3'; reverse, 5'-gtgatccagcaggtggaagt-3', TIGAR-forward, 5'-attca gacagcggtattccag-3'; reverse, 5'-aagcaccgtgactcacaactaa-3'. Human 18s RNA was used as the housekeeping control and amplified with the following primers: forward, 5'-cgattggatgg tttagtgagg-3'; reverse, 5'-agttcgaccgtcttctcagc-3'. Taq DNA polymerase was used to amplify the PCR product with 55 cycles at $95^{\circ} \mathrm{C}$ for $15 \mathrm{sec}$, at $60^{\circ} \mathrm{C}$ for $30 \mathrm{sec}$, and at $45^{\circ} \mathrm{C}$ for $30 \mathrm{sec}$.

Western blot analysis. Western blot analysis was performed as previously described (21) using corresponding antibodies against p53 (Santa Cruz, CA, USA), B-actin (Sigma), MnSOD (Upstate, MA, USA), or GPx1 (Abcam, MA, USA). Representative blots and quantification from three independent experiments are shown.

Statistical analysis. Quantitative results are expressed as mean \pm SEM. Statistical analysis was performed using Oneway ANOVA for multiple-group comparison.

\section{Results}

$2 D G$ reduces ATP production of p53-defective cells but not p53-wt cells. Previously, we showed the differential cytotoxicity of the glycolytic inhibitor, 2DG, on non-small cell lung cancer (NSCLC) cells (21). p53-defective lung cancer cells, H358, are more sensitive to 2DG than p53-wt A549 cells, as demonstrated by clonogenic survival assay. In this study, we extend the initial observation to investigate how 2DG differentially kills cancer cells expressing different p53 status. First, we evaluated the effect of p53 on the glycolytic inhibitory effect of 2DG by measuring the levels of glucose in cultured medium after $20 \mathrm{mM}$ 2DG treatment. The presence of 2DG reduced the glucose uptake in A549 cells by $48 \mathrm{~h}$. Interestingly, glucose consumption of H358 
A

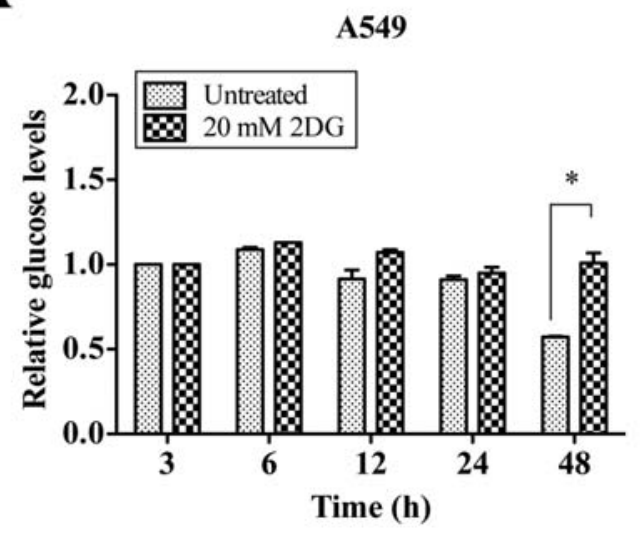

B

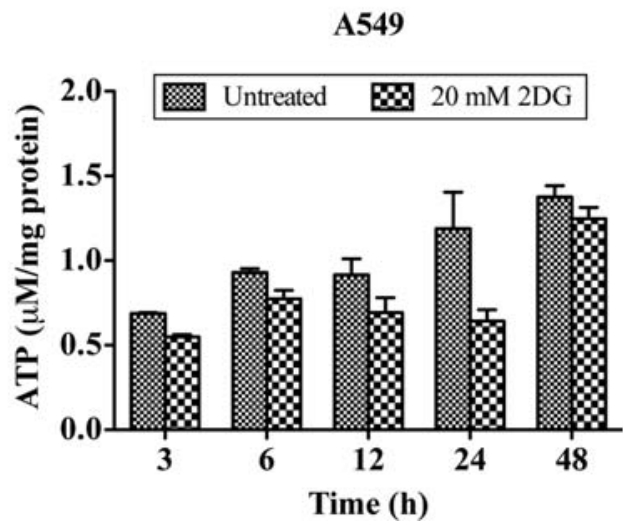

H358

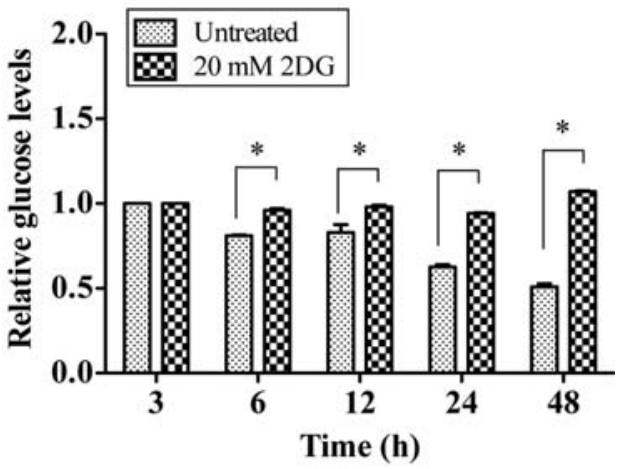

H358

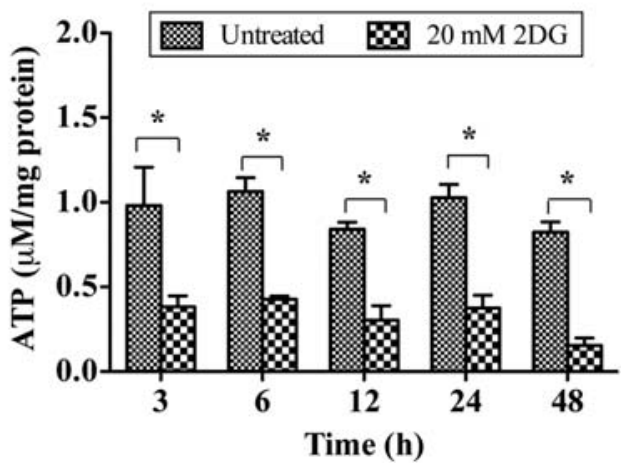

Figure 1.2DG reduces ATP production of p53-defective cells but not p53-WT cells. (A) The relative glucose levels in cultured medium. A549 and H358 cells were treated with 2DG for indicated periods of time. Cultured mediums were collected and processed for assay. (B) The levels of intracellular ATP. Cells were collected and homogenates were prepared for ATP determination. The amounts of cellular ATP were calculated and normalized to protein concentration. ${ }^{*} \mathrm{p}<0.05$ compared with corresponding control groups.

cells was significantly decreased as early as $6 \mathrm{~h}$ of $2 \mathrm{DG}$ treatment, indicating higher glucose utilization in $\mathrm{H} 358$ cells (Fig. 1A). These data are consistent with the regulatory role of p53 on glucose metabolism. To confirm the effect of 2DG on glucose consumption, we further determined the production of cellular energy, ATP, after 2DG treatment. Compared to the untreated control, 2DG significantly decreased the ATP levels of $\mathrm{H} 358$ cells as early as $3 \mathrm{~h}$ of treatment and reductions were maintained throughout the entire experimental period (Fig. 1B). The levels of ATP in 2DG-treated A549 cells, however, did not show any significant decrease and were comparable to levels of control cells at $48 \mathrm{~h}$ after treatment. These data indicate that p53 deficiency results in an increase of glycolysis with consequent higher sensitivity to $2 \mathrm{DG}$.

p53-defective cells are unable to adapt to 2DG-induced metabolic stress. As cellular ATP level is maintained through either glycolysis or OXPHOS, it is possible that 2DG may interfere with ATP production through glycolysis and/or OXPHOS. To address this question, we measured the concentration of lactate, the metabolite of the glycolytic pathway, and the activities of mitochondrial respiratory complexes following $20 \mathrm{mM}$ of $2 \mathrm{DG}$ treatment. Fig. 2A shows that $48 \mathrm{~h}$ of 2DG exposure decreased the lactate levels of A549 and H358 cells dose dependently compared to the untreated controls. The activities of mitochondrial complex I in both A549 (24, 48 h) and H358 cells (48 h) were significantly increased compared to the untreated control group (Fig. 2B). 2DG significantly increased complex II activities of A549 (3, $6 \mathrm{~h}$ ) and $\mathrm{H} 358$ cells (48 h) (Fig. 2C). These results suggest that p53 does not regulate the activities of mitochondrial respiratory complexes I and II in response to glycolytic inhibition by 2DG. In contrast, complex IV activity in A549 cells significantly increased at $48 \mathrm{~h}$ of 2DG treatment (Fig. 2D), whereas H358 cells did not show any change in complex IV activity, suggesting a lack of adaptive response via complex IV in p53-deficient cells.

$2 D G$ causes oxidative stress in lung cancer cells. Because mitochondria are major sites of ROS generation (23), it is possible that an increase in mitochondrial activity may facilitate the flow of electrons down the respiratory chain leading to increased ROS production. During the respiration process, electrons reduce molecular oxygen to produce superoxide radicals and can escape from the electron transport chain (23). Thus, we used the levels of mitochondrial superoxide $\left(\mathrm{O}_{2}{ }^{-}\right)$and intracellular reactive oxygen species (ROS) as indicators of the increase in mitochondrial respiratory activity and oxidative stress. A significant increase in mitochondrial $\mathrm{O}_{2}{ }^{-}$levels of A549 and $\mathrm{H} 358$ cells was 
A

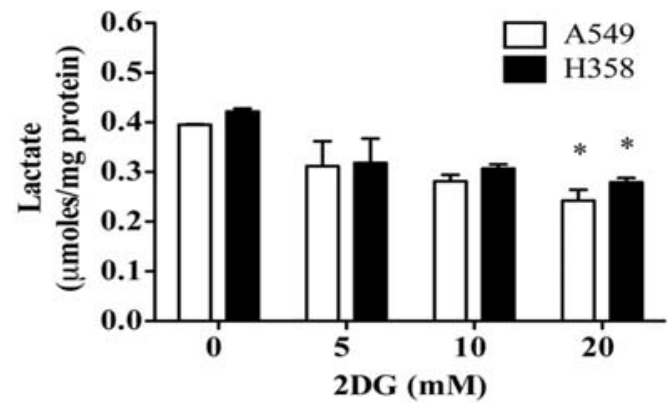

B

A549

H358
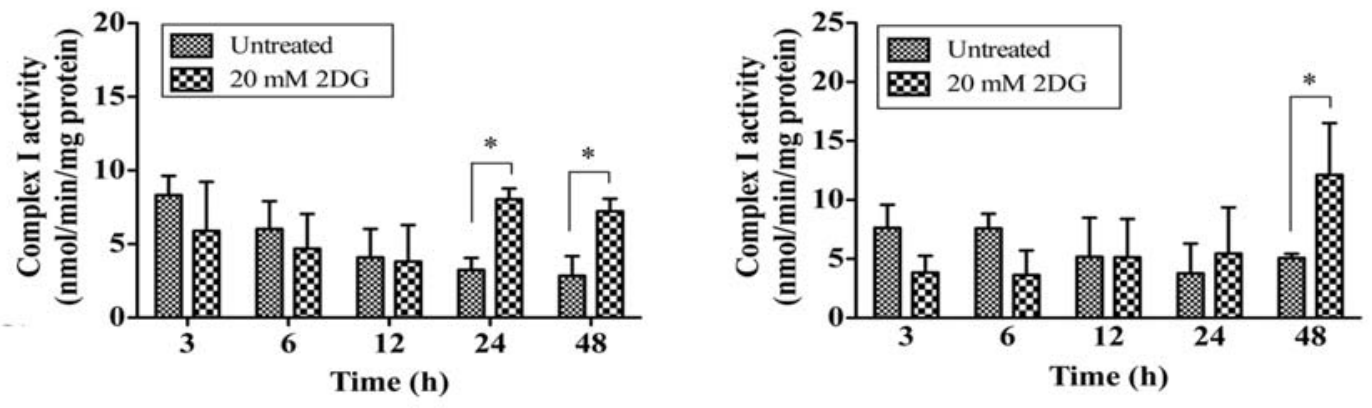

C

A549

H358
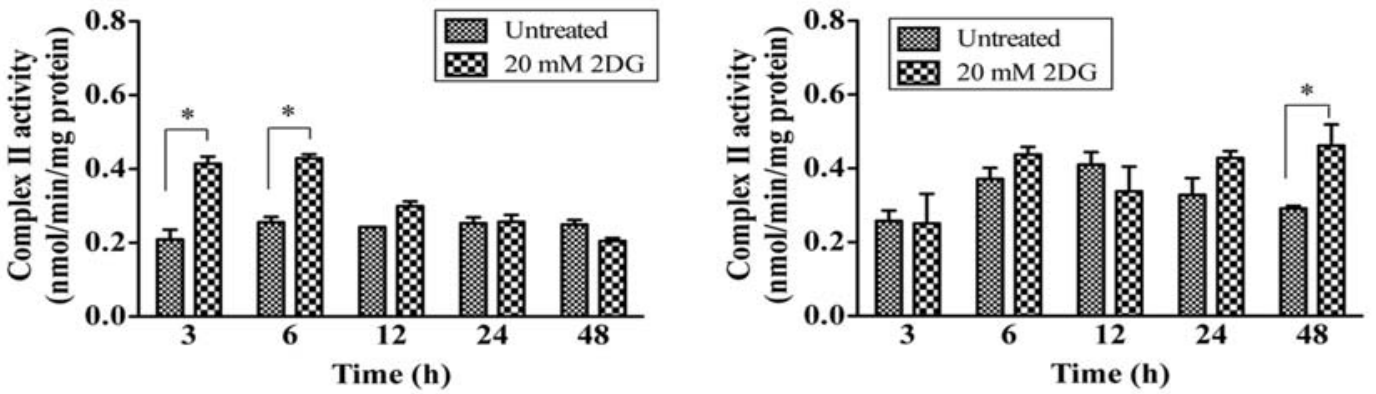

D

A549

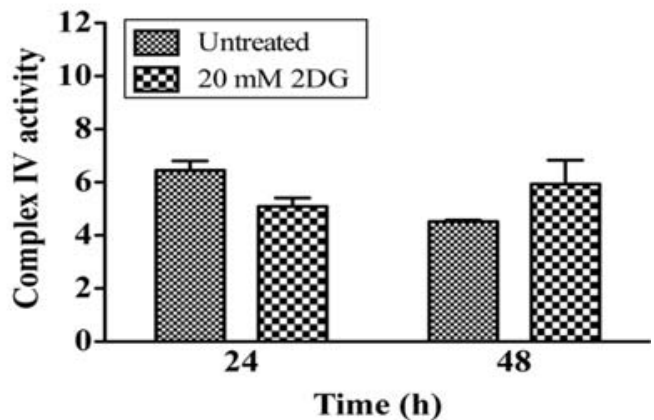

Figure 2. p53-defective cells are unable to adapt to 2DG-induced metabolic stress. (A) Lactate assay. 2DG was applied to A549 and H358 cells for 48 h. Cells were collected and processed for lactate determination. The amounts of lactate were normalized to protein levels. (B) The mitochondrial complex I activity assay. (C) The activity of mitochondrial complex II. (D) The activity of mitochondrial complex IV. Cells were collected and 25-50 $\mu \mathrm{g}$ of whole cell homogenates were used for measurement of enzymatic activities. " $\mathrm{p}<0.05$ compared with untreated control.

observed in 2DG-treated groups at $48 \mathrm{~h}$ (Fig. 3A). In addition, 2DG increased ROS levels significantly, 2- to 4fold, in both cell lines at 24 and $48 \mathrm{~h}$ of treatment compared to the untreated group (Fig. 3B). These results are consistent with an increase of OXPHOS in both A549 and H358 cells. p53 regulates oxidative stress responses to $2 D G$-induced oxidative stress. Oxidative stress is an imbalance between pro-oxidants and antioxidants. We therefore measured the levels of p53 targets, antioxidant enzymes in mitochondria, manganese superoxide dismutase (MnSOD) and glutathione peroxidase 1 (GPx1). The protein level of MnSOD, a mito- 
A

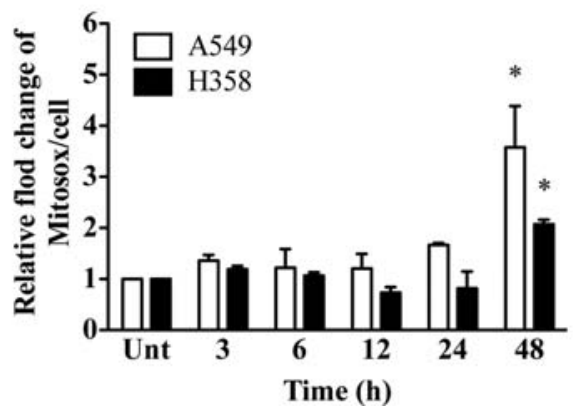

B

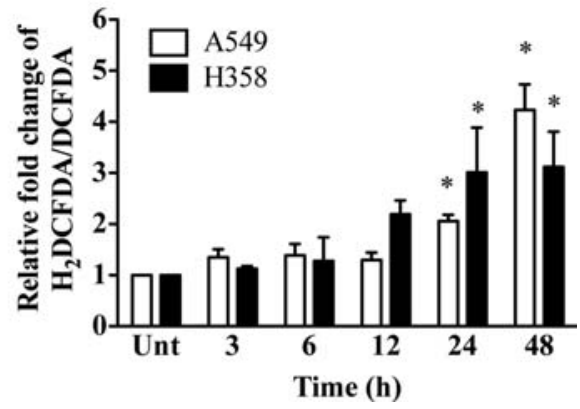

Figure 3. 2DG causes oxidative stress in lung cancer cells. (A) Mitochondrial superoxide measurement. Cells were incubated with mitoSOX (superoxide specific probe) after treatment with $20 \mathrm{mM}$ of $2 \mathrm{DG}$ for indicated time. The obtained fluorescences were normalized to number of cells. (B) Measurement of intracellular reactive oxygen species (ROS). Cells were incubated with carboxy- $\mathrm{H}_{2}$ DCFDA (ROS-sensitive probe) or carboxy-DCFDA (ROS-insensitive probe); then, the ratio of carboxy- $\mathrm{H}_{2}$ DCFDA to carboxy-DCFDA was compared. ${ }^{*} \mathrm{p}<0.05$ compared with no $2 \mathrm{DG}$ control groups. Unt, untreated control.

A

A549

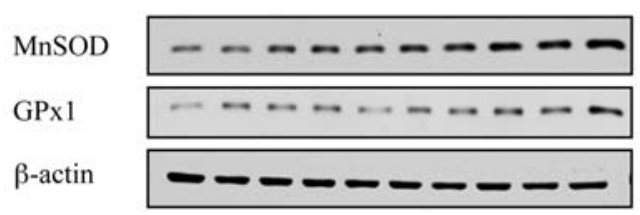

Time (h)

$20 \mathrm{mM} 2 \mathrm{DG}$

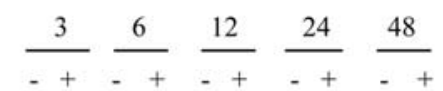

B
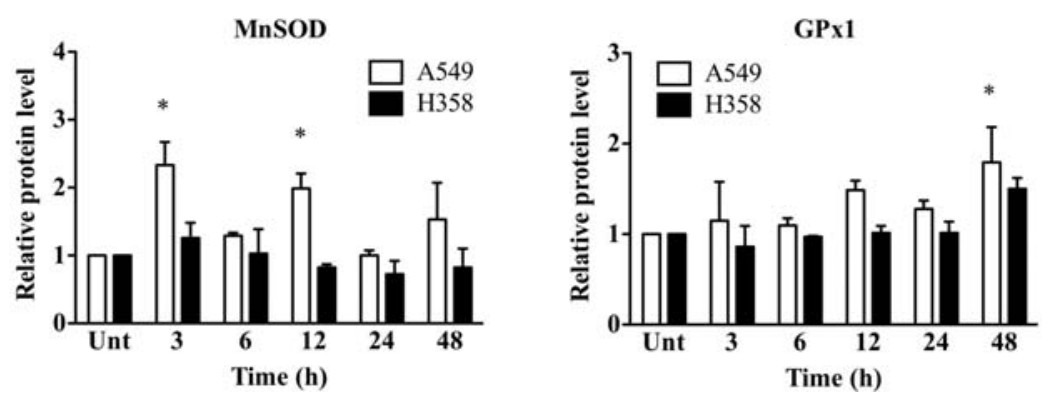

Figure 4. p53 regulates oxidative stress responses to 2DG-induced oxidative stress. (A) Representative Western blots showing the protein levels of MnSOD, GPx1. Cells were treated with or without $20 \mathrm{mM}$ of 2DG at designated time. Cells were then collected and processed for Western blot analysis. B-actin was probed as a loading control. (B) Quantitative analysis. * $\mathrm{p}<0.05$ compared with no 2DG control groups. Unt, untreated control.

chondrial localized antioxidant enzyme, was significantly increased in A549 cells as early as $3 \mathrm{~h}$ and $12 \mathrm{~h}$ after 2DG treatment (Fig. 4). The protein level of GPx1 significantly increased at $48 \mathrm{~h}$. Interestingly, the protein levels of GPx 1 and MnSOD were not significantly changed in H358 cells. These data show that p53 plays an important role in maintaining the levels of cellular antioxidant enzymes MnSOD and GPx1, which are important defenses against oxidative stress in mitochondria.

2DG-mediated oxidative stress induces activation of p53. Because p53 is an oxidative stress-responsive, tumorsuppressor protein, we determined the protein level of $\mathrm{p} 53$ after 2DG treatment. 2DG significantly increased the level of p53 protein at $48 \mathrm{~h}$ (Fig. 5A). The increase in p53 activity was verified by the mRNA level of p53 target genes using quantitative RT-PCR. The mRNA levels of the synthesis of cytochrome c oxidase 2 (SCO2) were significantly increased at 6 and $12 \mathrm{~h}$, and the mRNA levels of TP53-induced glycolysis and the apoptosis regulator (TIGAR) increased significantly at 3 and $6 \mathrm{~h}$ after $2 \mathrm{DG}$ treatment (Fig. 5B). Thus, p53 may regulate metabolic response to 2DG by upregulating its target genes.

\section{Discussion}

It has been demonstrated that 2DG inhibits glycolysis and energy production in several types of cancer cells (24). We 
A

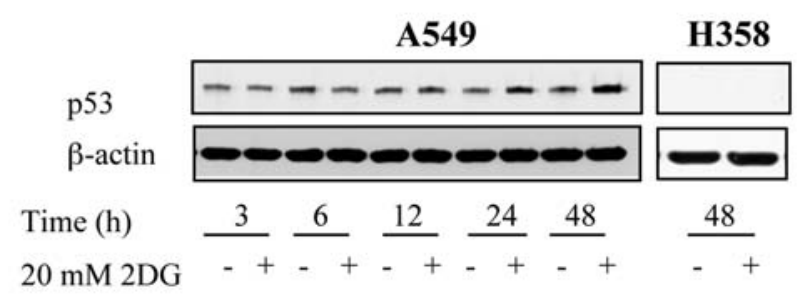

p53

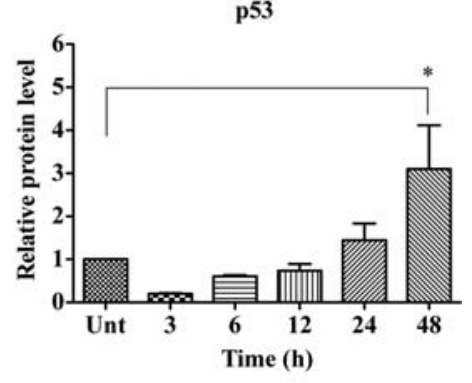

B
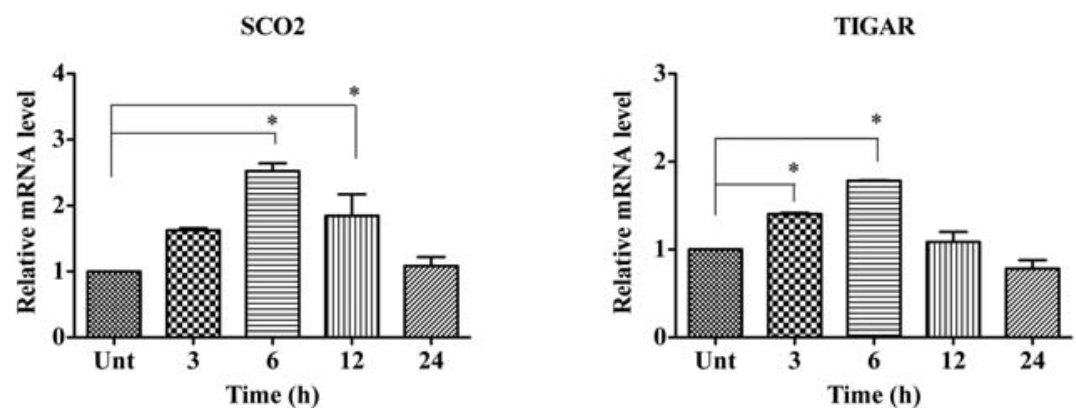

Figure 5. 2DG-mediated oxidative stress induces activation of p53. (A) The protein levels of p53. After incubation with 20 mM $2 \mathrm{DG}$ for indicated time, cells were collected and processed for Western blot analysis. (B) The relative mRNA levels of SCO2 and TIGAR. The levels of mRNA were quantified by realtime RT-PCR. ${ }^{*}$ p $<0.05$ compared with untreated control. Unt, untreated control.

previously showed that p53 plays an important role in 2DGinduced cytotoxicity because the presence of p53 reduces the cytotoxic effect of 2DG (21). In the present study, we reveal the mechanisms by which p53 mediates a protective effect against glycolytic inhibition. Our data show that 2DG significantly decreases glucose consumption in both A549 and H358 cells (Fig. 1A). However, the glycolysis inhibitory effect of 2DG was more pronounced in H358 cells, supporting the role of p53 in glycolytic regulation (25). Notably, the ATP levels in p53-wt cells (A549) were unaltered by $2 \mathrm{DG}$ in contrast to the significant decrease in p53-deficient cells (H358) (Fig. 1B), which is consistent with our previous work demonstrating the higher susceptibility of p53-deficient cells to 2DG (21). 2DG decreases the levels of lactate, a glycolytic metabolite in both A549 and H358 cells (Fig. 2A). Cells containing wild-type p53 successfully compensated for 2DG-inhibited glycolysis through enhanced oxidative phosphorylation as evidenced by unchanged ATP levels and induction of mitochondrial respiratory complexes. In contrast, p53-defective cells exhibited a failure to maintain ATP levels due to defective mitochondrial cytochrome c oxidase (complex IV) function (Figs. 1B and 2B-D). Our data support the concept that $\mathrm{p} 53$-defective cells are more glycolytic-dependent than p53 wild-type cells are $(26,27)$ and are consistent with the finding that the cytotoxic effect of $2 \mathrm{DG}$ is greater in cancer cells exhibiting hypoxic or mitochondrial respiratory defects (28-30).

Mitochondrial respiration is the main source of reactive oxygen species (ROS), especially superoxide, in cells (23). It is estimated that $1-2 \%$ of total molecular oxygen utilized by mammalian mitochondria is converted into superoxide anion (31). Our data show that 2DG-modulated mitochondrial complex activities generate more mitochondrial superoxide and intracellular ROS levels (Fig. 3). In A549 cells, the induction of activities of mitochondrial complex I and complex IV $(24,48 \mathrm{~h})$ was accompanied by increased mitochondrial superoxide $(48 \mathrm{~h})$ and intracellular ROS $(24,48 \mathrm{~h})$, suggesting that the generation of mitochondrial ROS is due mainly to an increase in complex I activity. In $\mathrm{H} 358$ cells, the increase in mitochondrial ROS at $48 \mathrm{~h}$ was accompanied by an increase in complexes I and II activities. Our findings reveal that 2DG induced mitochondrial oxidative stress in A549 and H358 cells through the induction of OXPHOS, consistent with the induction of oxidative stress in other cancer cells types including cervix, colon, head and neck, and breast (32-34).

Interestingly, we discovered that the mitochondrial oxidative stress in $\mathrm{H} 358$ cells not only results from mitochondrial complex activities that induce pro-oxidants, but also from a lack of adaptive response in the antioxidant system, as significant induction of MnSOD and GPx1 was observed in A549 cells but not in H358 cells (Fig. 4). The expression of MnSOD and GPx 1 has been shown to be transcriptionally upregulated by p53 (35). Thus, it is possible that the induction of antioxidants protects against 2DGinduced oxidative stress in p53-positive, A549 cells, and the observed enhanced sensitivity of H358 cells may result from the lack of increased protection against oxidative stress caused by $2 \mathrm{DG}$.

2DG consistently and significantly induces the level of stress-responsive tumor suppressor p53 protein in A549 cells, which is accompanied by increases in the activities of mitochondrial respiratory complexes and ROS levels, suggesting that elevation of $\mathrm{p} 53$ is mediated by mitochondrial 
ROS (Fig. 5A). Induction of mRNA levels of the p53 targets, SCO2 and TIGAR, was observed, confirming the transcriptional function of p53 under metabolic stress conditions induced by 2DG in A549 cells (Fig. 5B). SCO2 encodes a copper chaperone protein required for the assembly of mitochondrial cytochrome c oxidase complex IV (8). Thus, p53 induction of mRNA expression of $\mathrm{SCO} 2$ may enhance OXPHOS, which results in maintenance of energy (ATP) and survival of A549 cells. This possibility is supported by a recent study demonstrating that $\mathrm{SCO} 2^{-/-}$human colon cancer cells exhibit defective oxygen consumption and higher sensitivity to $2 \mathrm{DG}$ than $\mathrm{SCO} 2^{+/+}$cells do (36).

Together, our data suggest that p53 confers resistance to the glycolytic inhibitor 2DG through: i) compensation of glycolytic inhibition by the induction of OXPHOS, ii) activation of p53 on metabolic targets, and iii) induction of antioxidant response. Our findings show that 2DG inhibition of glycolysis is effective in cancer with defective p53, which accounts for more than $50 \%$ of all types of cancer (37). This information is important for the development of a novel therapeutic strategy designed to alter metabolic activity for selectively killing cancer cells.

\section{Acknowledgements}

This work was supported by NIH grants CA 115801, CA 049797, and the Royal Golden Jubilee (RGJ) program, Thailand Research Fund, Thailand.

\section{References}

1. Warburg O: On the origin of cancer cells. Science 123: 309-314, 1956.

2. Weber G: Enzymology of cancer cells (second of two parts). N Engl J Med 296: 541-551, 1977.

3. Pfeiffer T, Schuster S and Bonhoeffer S: Cooperation and competition in the evolution of ATP-producing pathways. Science 292: 504-507, 2001.

4. Gillies RJ and Gatenby RA: Adaptive landscapes and emergent phenotypes: why do cancers have high glycolysis? J Bioenerg Biomembr 39: 251-257, 2007.

5. Koukourakis MI, Giatromanolaki A, Harris AL and Sivridis E: Comparison of metabolic pathways between cancer cells and stromal cells in colorectal carcinomas: a metabolic survival role for tumor-associated stroma. Cancer Res 66: 632-637, 2006.

6. Bensaad K and Vousden KH: p53: new roles in metabolism. Trends Cell Biol 17: 286-291, 2007.

7. Matoba S, Kang JG, Patino WD, et al: p53 regulates mitochondrial respiration. Science 312: 1650-1653, 2006.

8. Jaksch M, Paret C, Stucka R, et al: Cytochrome c oxidase deficiency due to mutations in SCO2, encoding a mitochondrial copper-binding protein, is rescued by copper in human myoblasts. Hum Mol Genet 10: 3025-3035, 2001.

9. Okamura S, Ng CC, Koyama K, et al: Identification of seven genes regulated by wild-type p53 in a colon cancer cell line carrying a well-controlled wild-type p53 expression system. Oncol Res 11: 281-285, 1999.

10. Tian WN, Braunstein LD, Apse K, et al: Importance of glucose6-phosphate dehydrogenase activity in cell death. Am J Physiol 276: C1121-C1131, 1999

11. Lyakhov IG, Krishnamachari A and Schneider TD: Discovery of novel tumor suppressor p53 response elements using information theory. Nucleic Acids Res 36: 3828-3833, 2008.

12. Bensaad K, Tsuruta A, Selak MA, et al: TIGAR, a p53-inducible regulator of glycolysis and apoptosis. Cell 126: 107-120, 2006.

13. Schwartzenberg-Bar-Yoseph F, Armoni M and Karnieli E: The tumor suppressor p53 down-regulates glucose transporters GLUT1 and GLUT4 gene expression. Cancer Res 64: 2627-2633, 2004.

14. Kondoh H, Lleonart ME, Gil J, et al: Glycolytic enzymes can modulate cellular life span. Cancer Res 65: 177-185, 2005.
15. Jain VK, Kalia VK, Gopinath PM, Naqvi S and Kucheria K: Optimization of cancer therapy: part III-effects of combining 2deoxy-D-glucose treatment with gamma-irradiation on normal mice. Indian J Exp Biol 17: 1320-1325, 1979.

16. Kalia VK, Jain VK and Otto FJ: Optimization of cancer therapy: part IV-effects of 2-deoxy-D-glucose on radiation induced chromosomal damage in PHA-stimulated peripheral human leukocytes. Indian J Exp Biol 20: 884-888, 1982.

17. Singh SP, Singh S and Jain V: Effects of 5-bromo-2-deoxyuridine and 2-deoxy-D-glucose on radiation-induced micronuclei in mouse bone marrow. Int J Radiat Biol 58: 791-797, 1990.

18. Swamy RK, Manickam J, Adhikari JS and Dwarakanath BS: Glycolytic inhibitor, 2-deoxy-D-glucose, does not enhance radiation-induced apoptosis in mouse thymocytes and splenocytes in vitro. Indian J Exp Biol 43: 686-692, 2005.

19. Zhang XD, Deslandes E, Villedieu M, et al: Effect of 2-deoxyD-glucose on various malignant cell lines in vitro. Anticancer Res 26: 3561-3566, 2006.

20. Aft RL, Zhang FW and Gius D: Evaluation of 2-deoxy-Dglucose as a chemotherapeutic agent: mechanism of cell death Br J Cancer 87: 805-812, 2002.

21. Sinthupibulyakit C, Grimes KR, Domann FE, et al: p53 is an important factor for the radiosensitization effect of 2-deoxy-Dglucose. Int J Oncol 35: 609-615, 2009.

22. Yen HC, Oberley TD, Gairola CG, Szweda LI and St Clair DK: Manganese superoxide dismutase protects mitochondrial complex I against adriamycin-induced cardiomyopathy in transgenic mice. Arch Biochem Biophys 362: 59-66, 1999.

23. Turrens JF: Mitochondrial formation of reactive oxygen species. J Physiol 552: 335-344, 2003.

24. Dwarkanath BS, Zolzer F, Chandana S, et al: Heterogeneity in 2-deoxy-D-glucose-induced modifications in energetics and radiation responses of human tumor cell lines. Int J Radiat Oncol Biol Phys 50: 1051-1061, 2001.

25. Cheung EC and Vousden KH: The role of p53 in glucose metabolism. Curr Opin Cell Biol 22: 186-191, 2010.

26. Ibrahim MM, Razmara M, Nguyen D, Donahue RJ, Wubah JA and Knudsen TB: Altered expression of mitochondrial 16S ribosomal RNA in p53-deficient mouse embryos revealed by differential display. Biochim Biophys Acta 1403: 254-264, 1998.

27. Zhou S, Kachhap S and Singh KK: Mitochondrial impairment in p53-deficient human cancer cells. Mutagenesis 18: 287-292, 2003.

28. Maher JC, Krishan A and Lampidis TJ: Greater cell cycle inhibition and cytotoxicity induced by 2-deoxy-D-glucose in tumor cells treated under hypoxic vs aerobic conditions. Cancer Chemother Pharmacol 53: 116-122, 2004.

29. Liu H, Hu YP, Savaraj N, Priebe W and Lampidis TJ: Hypersensitization of tumor cells to glycolytic inhibitors. Biochemistry 40: 5542-5547, 2001

30. Liu H, Savaraj N, Priebe W and Lampidis TJ: Hypoxia increases tumor cell sensitivity to glycolytic inhibitors: a strategy for solid tumor therapy (Model C). Biochem Pharmacol 64: 1745-1751, 2002.

31. Cadenas E and Davies KJ: Mitochondrial free radical generation, oxidative stress, and aging. Free Radic Biol Med 29: 222-230, 2000.

32. Lin X, Zhang F, Bradbury CM, et al: 2-Deoxy-D-glucoseinduced cytotoxicity and radiosensitization in tumor cells is mediated via disruptions in thiol metabolism. Cancer Res 63: 3413-3417, 2003.

33. Aykin-Burns N, Ahmad IM, Zhu Y, Oberley LW and Spitz DR: Increased levels of superoxide and $\mathrm{H}_{2} \mathrm{O}_{2}$ mediate the differential susceptibility of cancer cells versus normal cells to glucose deprivation. Biochem J 418: 29-37, 2009.

34. Simons AL, Ahmad IM, Mattson DM, Dornfeld KJ and Spitz DR: 2-Deoxy-D-glucose combined with cisplatin enhances cytotoxicity via metabolic oxidative stress in human head and neck cancer cells. Cancer Res 67: 3364-3370, 2007.

35. Hussain SP, Amstad P, He P, et al: p53-induced up-regulation of MnSOD and GPx but not catalase increases oxidative stress and apoptosis. Cancer Res 64: 2350-2356, 2004.

36. Ho Joong Sung WM, Wang P-Y, Hynes J, O'Riordan TC, Combs CA, McCoy JP Jr, Bunz F, Kang J-G and Hwang PM: Mitochondrial respiration protects against oxygen-associated DNA damage. Nat Commun (In press).

37. Soussi T and Beroud C: Assessing TP53 status in human tumours to evaluate clinical outcome. Nat Rev Cancer 1: 233-240, 2001. 